\section{Avaliação do impacto de intervenção educativa em farmácias com promoção comercial de produtos que competem com 0 aleitamento materno}

\section{Impact assessment of an educational intervention in pharmacies that use commercial promotion of products competing with maternal breastfeeding}

\author{
Evaluación del impacto de una intervención \\ educativa en farmacias con promoción \\ comercial de productos que compiten \\ con la lactancia materna
}

\author{
Gloria Priscila Nunes Rodrigues 1 \\ Maria Inês Couto de Oliveira ${ }^{2}$ \\ Cristiano Siqueira Boccolini 3 \\ Enilce de Oliveira Fonseca Sally 4 \\ José Rodrigo de Moraes 5
}

doi: 10.1590/0102-311X00129919

\title{
Resumo
}

A Norma Brasileira de Comercialização de Alimentos para Lactentes e Crianças de Primeira Infância, Bicos, Chupetas e Mamadeiras (Lei Federal no 11.265/2006) visa controlar o marketing abusivo de produtos que competem com o aleitamento materno. O objetivo foi avaliar o impacto de uma intervenção educativa sobre o cumprimento da Lei em farmácias. Pesquisa de intervenção randomizada conduzida em 155 farmácias que infringiam a Lei na Zona Sul do Rio de Janeiro, Brasil. A prática comercial das farmácias foi avaliada antes e depois da intervenção com o farmacêutico e com o gerente, comparados ao grupo controle. O intervalo entre a intervenção e a segunda avaliação foi de um mês. Para aferir as mudanças no quantitativo de farmácias com infração à Lei, antes e depois da intervenção, foi utilizado o teste de McNemar. O teste de Wilcoxon foi usado para comparar a variação no número de infrações em cada grupo de alocação. Um mês após a intervenção houve redução de $16,1 \%(n=25)$ no total de farmácias com infração. Houve redução de $18,7 \%$ para $12,9 \%$ em produtos cuja promoção comercial é proibida $(p=0,093)$ e de $92,9 \%$ para $80,5 \%$ nos produtos lácteos $(p=0,001)$, mas entre alimentos de transição houve aumento de 28,5\% para 42,3\% de farmácias com promoção ilegal $(p=0,006)$. O grupo intervenção com farmacêutico apresentou redução estatisticamente significativa nas infrações relacionadas a descontos de preço $(p=0,022)$ e a exposições especiais $(p=0,002)$. A intervenção educativa reduziu a quantidade de farmácias que infringia essa Lei, principalmente quando esta intervenção foi realizada com o farmacêutico.

Estudos Experimentais; Aleitamento Materno; Legislação sobre Alimentos; Marketing; Substitutos do Leite Humano

\author{
Correspondência \\ M. I. C. Oliveira \\ Departamento de Epidemiologia e Bioestatística, Instituto de \\ Saúde Coletiva, Universidade Federal Fluminense. \\ Rua Marquês de Paraná 303, 3o andar - Prédio Anexo, Niteroi, \\ RJ 24030-210, Brasil. \\ marinescoutoliveira@gmail.com \\ 1 Programa de Pós-graduação em Saúde Coletiva, Universidade \\ Federal Fluminense, Niterói, Brasil. \\ 2 Instituto de Saúde Coletiva, Universidade Federal Fluminense, \\ Niterói, Brasil. \\ 3 Instituto de Comunicação e Informação Científica e \\ Tecnológica em Saúde, Fundação Oswaldo Cruz, Rio de Janeiro, \\ Brasil. \\ 4 Faculdade de Nutrição, Universidade Federal Fluminense, \\ Niterói, Brasil. \\ 5 Instituto de Matemática e Estatística, Universidade Federal \\ Fluminense, Niterói, Brasil.
}




\section{Introdução}

A Organização Mundial da Saúde (OMS) recomenda o aleitamento materno exclusivo até os seis meses de vida e a continuidade da amamentação até os dois anos de idade ou mais, complementada por outras fontes nutricionais ${ }^{1}$. Apesar dos seus reconhecidos benefícios 2 , a prática do aleitamento materno em nosso país ainda está aquém da recomendada 3 . Diversos fatores contribuem para a baixa prevalência de aleitamento materno exclusivo, como a baixa escolaridade materna, o trabalho materno fora de casa, a falta de orientação no pré-natal, condutas hospitalares e suporte pós-parto inadequados 4 . A comercialização de fórmulas infantis e de produtos que competem com o aleitamento materno afeta de forma negativa a amamentação e fomenta um mercado de mais de 40 bilhões de dólares, indicando o grande poder econômico das indústrias para a geração de campanhas publicitárias abusivas e de promoções comerciais inadequadas de alimentos infantis 5 .

Com o objetivo de proteger as mães contra o marketing abusivo de produtos que competem com o aleitamento materno foram criadas políticas globais e locais de proteção à amamentação ${ }^{6}$. O Código Internacional de Comercialização de Substitutos do Leite Materno representa o mais importante marco mundial de proteção à amamentação, tendo sido lançado pela OMS em 1981 7. No Brasil, a Política Nacional de Promoção, Proteção e Apoio ao Aleitamento Materno é composta por oito eixos, sendo a Norma Brasileira de Comercialização de Alimentos para Lactentes e Crianças de Primeira Infância, Bicos, Chupetas e Mamadeiras (NBCAL) um instrumento legal fundamental no eixo da proteção à amamentação 8 .

A NBCAL foi criada em 1988, sendo aprimorada 9 até ser transformada na Lei Federal no 11.265/ 2006 10, a qual conceitua "promoção comercial" como o conjunto de atividades informativas e de persuasão realizado com o objetivo de induzir a aquisição ou venda de determinado produto. Essa Lei veda a promoção comercial de fórmulas infantis, bicos, chupetas e mamadeiras. Leites podem ser alvo de promoção comercial, desde que acompanhados da frase de advertência "O Ministério da Saúde informa: o aleitamento materno evita infecções e alergias e é recomendado até os dois anos ou mais", assim como os alimentos de transição, cuja promoção comercial deve vir acompanhada da frase "O Ministério da Saúde informa: após os seis meses de idade continue amamentando seu filho e ofereça novos alimentos" 10.

Investigação conduzida em uma região do Rio de Janeiro em 201711 observou que 64,2\% das farmácias não cumpriam a NBCAL e a maior parte dos responsáveis por estes estabelecimentos desconhecia a Lei no 11.265/2006. Como o Brasil dispõe de uma legislação avançada de proteção à amamentação, porém pouco divulgada, praticada e fiscalizada, o presente estudo teve como objetivo avaliar o impacto de uma intervenção educativa sobre o cumprimento da NBCAL em farmácias. Avaliar consiste em emitir um juízo de valor sobre uma intervenção, fornecendo informações cientificamente válidas para que os diferentes atores envolvidos tenham condições de construir um julgamento que possa se traduzir em ação 12 . A presente avaliação de intervenção educativa visou contribuir para o cumprimento da NBCAL.

\section{Métodos}

É um estudo experimental randomizado que incluiu todas as farmácias com infração à NBCAL localizadas na Zona Sul da cidade do Rio de Janeiro, área que abrange 18 bairros de diferentes perfis socioeconômicos, incluindo as favelas da Rocinha e Vidigal.

As unidades de análise foram as farmácias. Os produtos abrangidos pela NBCAL foram classificados em cinco categorias: (1) fórmulas infantis para lactentes e fórmulas infantis de seguimento para lactentes; (2) fórmulas infantis para lactentes com necessidades dietoterápicas específicas; (3) mamadeiras, bicos e chupetas; (4) leites fluidos ou em pó de origem animal ou vegetal e compostos lácteos; e (5) alimentos de transição.

A presença de promoção comercial dos produtos 1, 2 e 3 ou a promoção comercial de produtos 4 ou 5 , sem a devida frase de advertência, constitui infração à NBCAL, considerada como desfecho no presente estudo. A promoção comercial foi classificada como "desconto no preço" quando havia redução de preço, oferta em destaque, desconto cumulativo, programa de fidelização, cartão ou cupom de desconto; como "exposição especial" quando havia alocação do produto na vitrine, em ponta de gôndola, empilhamento dos produtos em forma de pirâmide, ilha ou outras estratégias de destaque 
do produto e como "brinde" quando algum item ou amostra grátis era oferecida ou disponibilizada em conjunto com produtos relacionados à NBCAL 10. Como alvos da intervenção educativa foram selecionados os gerentes e os farmacêuticos, por seus papéis na organização da comercialização de alimentos e produtos infantis nas farmácias.

O critério de inclusão da farmácia na pesquisa de intervenção educativa foi a presença de infração à NBCAL na comercialização de alimentos infantis, mamadeiras, bicos e/ou chupetas. O critério de exclusão foi o estabelecimento não ter registro no Cadastro Nacional da Pessoa Jurídica (CNPJ).

Uma pesquisa anterior, realizada em 2017, denominada Avaliação do cumprimento da Norma Brasileira de Comercialização de Alimentos para Lactentes e Crianças de Primeira Infância em Estabelecimentos Comerciais, abrangeu todas as farmácias, os supermercados e as lojas de departamentos localizados na Zona Sul da cidade do Rio de Janeiro, e identificou 240 farmácias que comercializavam alimentos e produtos infantis abrangidos pela NBCAL 11 .

Essas 240 farmácias foram listadas e aleatoriamente distribuídas em três grupos: grupo de intervenção com o farmacêutico (GIF), grupo de intervenção com o gerente comercial (GIG) e grupo controle (GC). Para alocar as farmácias em cada um dos três grupos foi aplicado o algoritmo de Hájek 13 , que consiste em gerar para cada farmácia um número aleatório uniformemente distribuído no intervalo $(0,1)$ e ordená-las em ordem crescente do número aleatório. As farmácias correspondentes aos $\mathrm{n} 1$ (menores números aleatórios) foram alocadas ao GIF; as correspondentes aos $\mathrm{n} 2$ (números aleatórios seguintes) foram alocadas ao GIG e as farmácias restantes ao GC.

Estimou-se que uma amostra de pelo menos 37 farmácias por grupo garantiria $90 \%$ de poder para detectar uma diferença esperada de no mínimo $25 \%$ na prevalência de infração entre cada grupo de intervenção e o GC, com nível de significância de 5\%. Como se desejava comparar cada grupo de intervenção (com o farmacêutico e com o gerente) com o GC, o tamanho total da amostra (mínimo) requerido seria de 111 farmácias. No entanto, optou-se por partir das 240 farmácias listadas e aleatoriamente distribuídas nos três grupos, pois estas seriam avaliadas para verificar a presença ou não de infrações à NBCAL, e apenas as que apresentassem inconformidade à Lei no 11.265/2006 participariam da pesquisa de intervenção educativa.

Este estudo foi financiado pelo Conselho Nacional do Desenvolvimento Científico e Tecnológico (CNPq; processo no 404948/2016-2) e aprovado pelo Comitê de Ética em Pesquisa (CEP) da Universidade Federal Fluminense (UFF; parecer no 2.580.398/2018). Foi autorizada pelo CEP a dispensa da anuência dos estabelecimentos comerciais para a realização da coleta de dados com vistas à avaliação do cumprimento da NBCAL. Grande parte das farmácias está organizada em rede 11, o que poderia ocasionar informação privilegiada sobre a ação educativa e enviesar os resultados. Foi realizado um estudo piloto para teste e aprimoramento do material educativo e do questionário e para a padronização do processo de coleta de dados pelos entrevistadores. Esse estudo ocorreu em dois locais distantes da Zona Sul, para evitar a contaminação das farmácias onde seria realizada a pesquisa.

O trabalho de campo foi conduzido por quatro profissionais da área da saúde, previamente capacitados em cursos da NBCAL promovidos pela Secretaria de Estado de Saúde do Rio de Janeiro (SES-RJ), que atuaram sob a supervisão dos pesquisadores responsáveis. Para a avaliação do cumprimento da NBCAL foi utilizado formulário adaptado do usado pela Rede Internacional em Defesa do Direito de Amamentar (IBFAN; http://www.ibfan.org.br) e do NetCode da OMS 14, acrescido de questões pertinentes ao objetivo do estudo e adequado para a coleta de dados por meio de aplicativo móvel. Optou-se pelo aplicativo MAGPI (https://www.magpi.com/), compatível com o dispositivo do tipo celular com sistemas operacionais da Apple e Android 15, o qual dispunha de interface intuitiva para a entrada de dados, hospedagem eletrônica nos servidores da Fundação Oswaldo Cruz (Fiocruz), acompanhamento online da coleta de dados, e possibilidade de exportação de dados para os pacotes estatísticos mais comuns. O formulário eletrônico continha questões relativas ao perfil da farmácia, aos produtos comercializados, ao cumprimento da NBCAL, ao perfil do entrevistado, ao seu conhecimento a respeito da NBCAL, à autonomia para definir preço e arrumação dos produtos e à visita de representantes comerciais de empresas de alimentos e produtos infantis à farmácia.

Foi elaborado um material didático padronizado, impresso a cores, ilustrado por diferentes imagens, com linguagem clara, para a utilização na intervenção educativa com gerentes e farmacêuticos. Esse material continha informações a respeito da importância da amamentação, dos objetivos da NBCAL, dos produtos abrangidos por esta Norma e dos principais tipos de promoção comercial. O 
material mostrava a forma adequada de comercializar os alimentos e produtos destinados a crianças menores de três anos, de acordo com a Lei no 11.265/2006.

Cada entrevistador recebeu uma cópia do material didático e um instrutivo para a realização padronizada da avaliação do cumprimento da NBCAL, da entrevista ao gerente ou ao farmacêutico e da intervenção educativa. Os entrevistadores foram designados previamente para coletar dados em bairros distintos, evitando a superposição da coleta.

Todas as farmácias que comercializavam produtos abrangidos pela NBCAL, inicialmente listadas, foram avaliadas pelos entrevistadores. Essa lista foi sendo atualizada na medida em que os entrevistadores percorriam as ruas dos respectivos bairros durante o trabalho de campo. Farmácias inexistentes no período da pesquisa foram excluídas e farmácias novas, que não constavam na lista, foram acrescentadas, sendo respeitada a seguinte ordem: GIF-GIG-GC, respectivamente.

O trabalho de campo ocorreu entre abril e junho de 2018 e constou de duas etapas. Na primeira, as farmácias eram avaliadas e aquelas que apresentavam infração à NBCAL eram incluídas na pesquisa. Foi estipulado um intervalo mínimo de 30 dias entre a primeira etapa e a segunda avaliação, para que as farmácias tivessem tempo para adequar suas práticas.

Ao chegar à farmácia, o entrevistador classificava o seu tamanho segundo a quantidade de gôndolas existentes. $\mathrm{O}$ estabelecimento era avaliado quanto à comercialização de produtos abrangidos pela NBCAL e quanto à presença de inconformidade à Lei no 11.265/2006. Caso fosse encontrada alguma irregularidade, a farmácia era selecionada para participar do estudo de intervenção, sendo então solicitada a presença do gerente ou farmacêutico, segundo o grupo de alocação prévia. O entrevistador se apresentava, explicava os objetivos da pesquisa, mostrava o parecer de aprovação do CEP e o Termo de Consentimento Livre e Esclarecido (TCLE) a ser assinado por ele, esclarecendo que a pesquisa não apresentava caráter punitivo, mas sim educativo e realizava a entrevista. Caso o gerente (ou farmacêutico) a ser entrevistado não estivesse presente no estabelecimento, o entrevistador era orientado a voltar à farmácia pelo menos duas vezes, se possível no mesmo dia ou na mesma semana, para a realização da entrevista.

Essa entrevista era realizada em local de escolha do entrevistado, sendo respeitados os critérios de sigilo e privacidade. Após a entrevista, nos grupos GIF e GIG procedia-se à leitura padronizada, clara e pausada do material educativo. A intervenção educativa teve uma duração média de dez minutos. $\mathrm{O}$ entrevistado tinha autonomia para interromper a intervenção, se fosse necessário. Ao final da intervenção educativa, o entrevistado era convidado a acompanhar o entrevistador até os pontos da farmácia onde havia promoção comercial em desacordo com a NBCAL. O entrevistado era estimulado a identificar as infrações à NBCAL e a refletir sobre como adequar o estabelecimento para o seu cumprimento, criando assim uma oportunidade para sanar possíveis dúvidas sobre a legislação vigente e reforçar a intervenção educativa. Ao final, o entrevistado recebia uma cópia da Lei no 11.265/2006, sendo solicitado que socializasse as informações recebidas junto aos demais responsáveis pela farmácia.

$\mathrm{Na}$ segunda etapa da pesquisa, o entrevistador retornava ao estabelecimento, mais uma vez avaliava o cumprimento da NBCAL e entrevistava o gerente ou farmacêutico, segundo o grupo de alocação. Em caso do entrevistado na primeira fase não estar mais trabalhando no estabelecimento, ou estar de férias, era substituído por profissional da mesma categoria. Ao final da entrevista os participantes dos três grupos de alocação receberam um fôlder educativo sobre a NBCAL, com conteúdo semelhante ao da intervenção educativa realizada na primeira etapa da pesquisa, reforçando a ação educativa e permitindo que os entrevistados do GC pudessem também ter acesso às informações sobre a Lei no 11.265/2006.

Ao final do trabalho de campo o banco de dados foi exportado para o programa SPSS (https:// www.ibm.com/). Inicialmente, foram realizadas análises dos dados relativos às características dos estabelecimentos comerciais, dos produtos comercializados e das estratégias promocionais utilizadas, e os resultados foram apresentados em forma de tabelas. O efeito da intervenção foi aferido comparando-se os resultados dos grupos GIF, GIG e GC quanto ao número de farmácias que infringiam a NBCAL e à quantidade de infrações encontradas em cada farmácia antes e após a intervenção.

O resultado foi aferido pelo cálculo da prevalência de farmácias com infração. Foi também comparada a diferença entre o número de infrações por estabelecimento nas duas fases da pesquisa por meio do teste não paramétrico de postos sinalizados de Wilcoxon 16. 
Em cada grupo de alocação foi contabilizado o número de farmácias não mais existentes em 2018 e as farmácias novas encontradas no trabalho de campo, bem como o quantitativo que infringia a NBCAL, incluído na pesquisa de intervenção.

As características das farmácias e de seus responsáveis na etapa 1 foram observadas segundo o grupo de alocação. Para observar se os grupos eram homogêneos, foram avaliadas diferenças entre os grupos GIF, GIG e GC por meio do teste qui-quadrado de Pearson $\left(\chi^{2}\right)$, com correção de Yates, utilizada quando a frequência esperada é inferior a 5, considerando o nível de significância de $5 \% 16$.

Calculou-se o quantitativo de farmácias que comercializava produtos abrangidos pela NBCAL e que a infringia, segundo a etapa da pesquisa e as categorias de produtos abrangidos pela NBCAL.

Utilizamos o teste de McNemar 16, para amostras dependentes, para aferir as mudanças no quantitativo de farmácias com infração à NBCAL da etapa 1 para a etapa 2, segundo o produto comercializado. As categorias de produtos 1, 2 e 3, cuja promoção comercial é proibida, foram agrupadas em um único tipo, e as categorias 4 e 5 foram analisadas como distintas. Foram classificadas como: "sem/sem" as farmácias onde não foi encontrada infração na categoria de produto nas duas etapas da pesquisa; "sem/com" aquelas onde foi encontrada infração apenas na segunda etapa; "com/sem" as farmácias onde foi encontrada infração apenas na primeira etapa e "com/com" aquelas onde foram encontradas infrações nas duas etapas da pesquisa. $\mathrm{O}$ mesmo teste foi usado para comparar a mudança no número de farmácias com infração à NBCAL entre as etapas 1 e 2, por grupo de alocação, segundo os diferentes tipos de estratégia comercial. Foi obtida a variação mediana do número de infrações à NBCAL por farmácia segundo o grupo de alocação, antes e após a intervenção educativa, bem como o desvio padrão e valores mínimos e máximos, e o teste não paramétrico de postos sinalizados de Wilcoxon foi utilizado para verificar se a variação foi significativa.

\section{Resultados}

Das 240 farmácias listadas pela pesquisa realizada em 2017 12, 35 haviam sido fechadas e 23 novas tinham sido abertas. Foram, portanto, encontradas 228 farmácias comercializando produtos abrangidos pela NBCAL na Zona Sul da cidade do Rio de Janeiro em 2018. Dessas, 68\% ( $\mathrm{n}=155)$ apresentaram algum tipo de infração à Lei no 11.265/2006 e foram selecionadas para a pesquisa de intervenção educativa: $32,2 \%(n=50)$ receberam intervenção educativa com o farmacêutico; $29,7 \%(n=46)$ intervenção educativa com o gerente e $38,1 \%(n=59)$ dos estabelecimentos fizeram parte do grupo controle (Figura 1).

A maioria $(\mathrm{n}=144 ; 92,9 \%)$ das 155 farmácias que participaram da pesquisa intervenção fazia parte de uma rede de estabelecimentos. As farmácias foram categorizadas como pequenas $(\mathrm{n}=49$; $31,6 \%)$, médias $(n=66 ; 42,6 \%)$ e grandes $(n=40 ; 25,8 \%)$. Na primeira etapa da pesquisa, houve seis recusas $(3,9 \%)$ à entrevista. A maioria dos entrevistados era do sexo feminino $(\mathrm{n}=101 ; 65,2 \%)$ e mais da metade $(\mathrm{n}=80 ; 53,7 \%)$ informou não conhecer a NBCAL. De acordo com a maior parte dos entrevistados, eles não possuíam autonomia suficiente para realizar a promoção comercial dos produtos, sendo necessária a autorização da "Central" (matriz), responsável por definir os preços $(83,9 \%)$ e a arrumação $(63,9 \%)$ dos produtos. A maioria $(\mathrm{n}=117 ; 78,5 \%)$ afirmou receber visita de representantes de indústrias de alimentos infantis, mamadeiras, bicos e/ou chupetas. Não foi encontrada diferença estatisticamente significativa $(\mathrm{p}<0,05)$ entre a distribuição dessas variáveis nos grupos intervenção com o farmacêutico, intervenção com o gerente e grupo controle, mostrando que a randomização gerou grupos comparáveis (Tabela 1).

Cerca de um quinto dos entrevistados $(19,4 \%)$ na primeira fase não estava mais trabalhando na farmácia, ou estava de férias na época da segunda entrevista, tendo sido substituídos por profissional da mesma categoria. A proporção de entrevistados que afirmou conhecer a NBCAL não foi alterada significativamente da primeira $(25,5 \%)$ para a segunda etapa $(26,5 \%)$ da pesquisa $(\mathrm{p}=0,565)$ (dados não mostrados em tabela).

Todas as farmácias avaliadas comercializavam fórmulas infantis, bem como leites/compostos lácteos, mas apenas 90,3\% $(\mathrm{n}=140)$ comercializavam fórmulas para necessidades dietoterápicas específicas. Bicos, chupetas e mamadeiras, assim como alimentos de transição, eram comercializados por 


\section{Figura 1}

Fluxograma da pesquisa intervenção educativa sobre a Norma Brasileira de Comercialização de Alimentos para Lactentes e Crianças de Primeira Infância, Mamadeiras, Bicos e Chupetas (NBCAL). Zona Sul da cidade do Rio de Janeiro, Brasil, 2018.

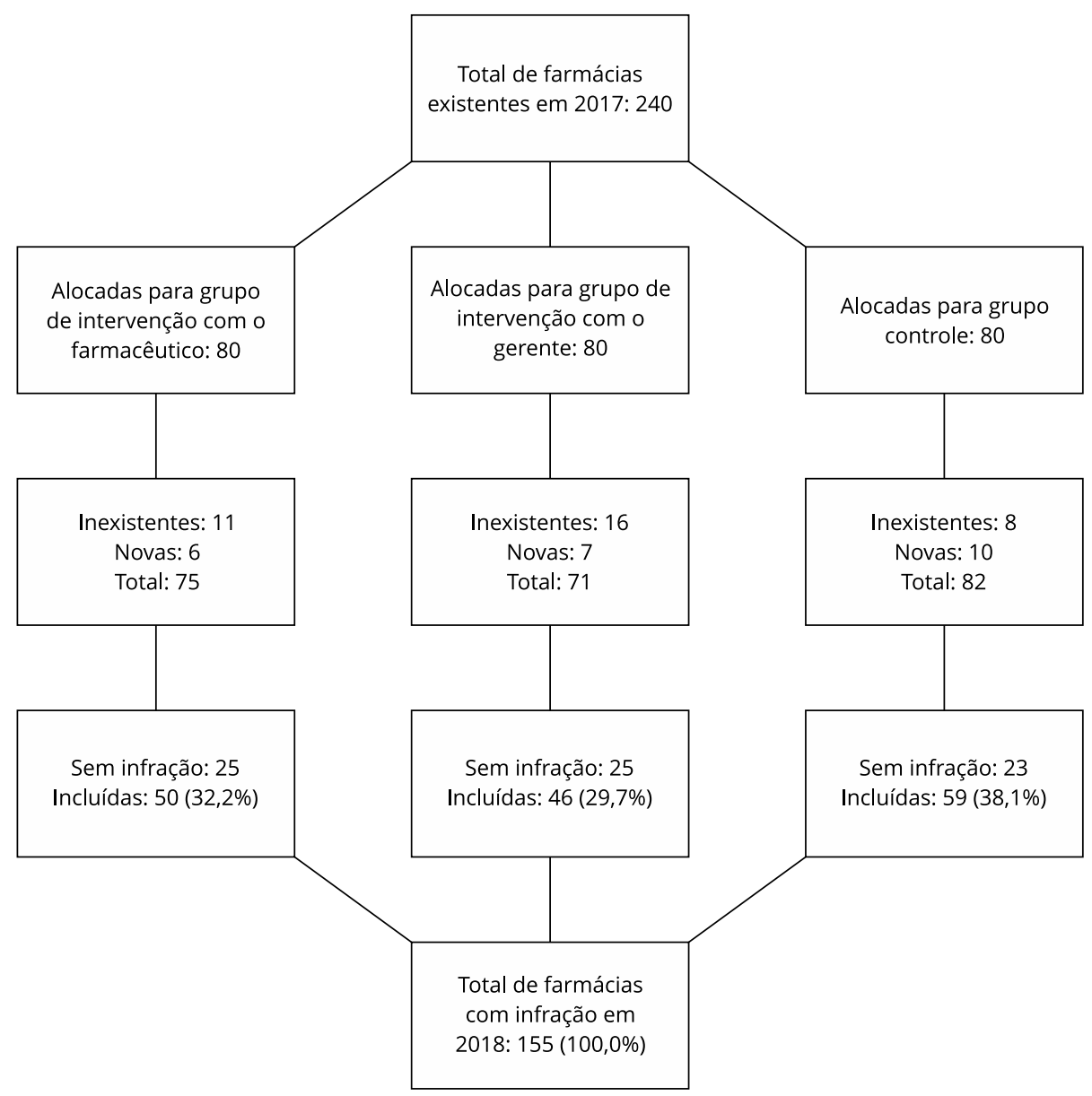

97,4\% ( $n=151)$ dessas farmácias. Na primeira etapa da pesquisa, 155 farmácias infringiam a NBCAL, este número reduziu para 130 (83,9\%) na segunda etapa (dados não mostrados em tabela).

Quanto ao tipo de produtos, foram encontradas 29 farmácias $(18,7 \%)$ com promoção comercial proibida pela Lei no 11.265/2006 antes da intervenção educativa (categorias com/sem e com/com), número que reduziu para $20(12,9 \%, \mathrm{p}=0,093)$ com promoção após a intervenção (categorias sem/ com e com/com). A proporção de famácias que promovia irregularmente leites/compostos lácteos reduziu de $92,3 \%(\mathrm{n}=143)$ para $80,5 \%(\mathrm{n}=124)(\mathrm{p}=0,001)$. No entanto, houve um aumento de $28,5 \%$ $(\mathrm{n}=42)$ para $42,3 \%(\mathrm{n}=63)$ naquelas que promoviam alimentos de transição em inconformidade com a Lei no $11.265 / 2006$ ( $\mathrm{p}=0,006$ ) (Tabela 2).

Nas farmácias cuja intervenção educativa foi realizada com o farmacêutico, 12\% deixaram de infringir a NBCAL na comercialização de produtos com promoção comercial proibida (categoria $\mathrm{com} / \mathrm{sem})$, e 4\% passaram a infringi-la (categoria sem/com) ( $\mathrm{p}=0,289)$. Na comercialização de leites/ compostos lácteos, 22\% deixaram de infringir a Lei e 4\% passaram a infringi-la, esta redução foi significativa e manteve a significância no total. Já na comercialização de alimentos de transição, 14,6\% deixaram de infringir a NBCAL e 16,7\% passaram a infringi-la, mas este aumento não foi estatisticamente significativo (Tabela 2). 
Tabela 1

Características das farmácias com algum tipo de infração à Norma Brasileira de Comercialização de Alimentos para Lactentes e Crianças de Primeira Infância, Bicos, Chupetas e Mamadeiras (NBCAL) e seus responsáveis na etapa 1, segundo grupo de alocação. Zona Sul da cidade do Rio de Janeiro, Brasil, 2018.

\begin{tabular}{|c|c|c|c|c|c|c|c|c|}
\hline \multirow[t]{3}{*}{ Características } & \multicolumn{6}{|c|}{ Grupo de alocação } & \multirow[t]{3}{*}{ Total } & \multirow[t]{3}{*}{ Valor de $p$} \\
\hline & \multicolumn{2}{|c|}{ GIF } & \multicolumn{2}{|c|}{ GIG } & \multicolumn{2}{|c|}{ GC } & & \\
\hline & $\mathbf{n}$ & $\%$ & $\mathbf{n}$ & $\%$ & $\mathbf{n}$ & $\%$ & & \\
\hline \multicolumn{9}{|c|}{ Farmácia faz parte de rede $(n=155)$} \\
\hline Sim & 45 & 90,0 & 45 & 97,8 & 54 & 91,5 & 144 & 0,287 \\
\hline Não & 5 & 10,0 & 1 & 2,2 & 5 & 8,5 & 11 & \\
\hline \multicolumn{9}{|c|}{ Tamanho da farmácia $(\mathrm{n}=155)$} \\
\hline Pequena & 15 & 30,0 & 12 & 26,1 & 22 & 37,3 & 49 & 0,758 \\
\hline Média & 22 & 44,0 & 20 & 43,5 & 24 & 40,7 & 66 & \\
\hline Grande & 13 & 26,0 & 14 & 30,4 & 13 & 22,0 & 40 & \\
\hline \multicolumn{9}{|c|}{ Sexo do entrevistado $(n=155)$} \\
\hline Feminino & 38 & 76,0 & 29 & 63,0 & 34 & 57,6 & 101 & 0,125 \\
\hline Masculino & 12 & 24,0 & 17 & 37,0 & 25 & 42,4 & 54 & \\
\hline \multicolumn{9}{|c|}{ Entrevistado conhece a NBCAL $(n=149)$} \\
\hline Sim & 13 & 27,1 & 9 & 21,4 & 16 & 27,1 & 38 & 0,933 \\
\hline Mais ou menos & 11 & 22,9 & 9 & 21,4 & 11 & 18,7 & 31 & \\
\hline Não & 24 & 50,0 & 24 & 57,2 & 32 & 54,2 & 80 & \\
\hline \multicolumn{9}{|c|}{ Farmácia recebe visita de representantes de empresas $(n=149)$} \\
\hline Sim & 36 & 75,0 & 38 & 90,5 & 43 & 72,9 & 117 & 0,081 \\
\hline Não & 12 & 25,0 & 4 & 9,5 & 16 & 27,1 & 32 & \\
\hline Total & 50 & 100,0 & 46 & 100,0 & 59 & 100,0 & 155 & \\
\hline
\end{tabular}

GC: grupo controle; GIF: grupo de intervenção com o farmacêutico; GIG: grupo de intervenção com o gerente.

Nas farmácias cuja intervenção educativa foi realizada com o gerente, 13\% deixaram de infringir a NBCAL na comercialização de fórmulas infantis, mamadeiras, bicos e chupetas e 6,5\% passaram a infringi-la. Na comercialização de leites/compostos lácteos, 8,9\% das farmácias deixaram de infringir a NBCAL e nenhuma passou a infringi-la. Essas diferenças, no entanto, não foram estatisticamente significantes. Já na comercialização de alimentos de transição, 6,8\% das farmácias deixaram de infringir a NBCAL, enquanto $38,6 \%$ passaram a infringí-la, um aumento que se manteve significativo para o total de farmácias (Tabela 2).

Nas farmácias do grupo controle, 6,8\% deixaram de infringir a NBCAL na comercialização de fórmulas infantis, bicos, mamadeiras e chupetas da primeira para a segunda etapa da pesquisa e 3,4\% passaram a infringí-la. Na comercialização de leites/compostos lácteos 15,3\% deixaram de infringir a NBCAL e 5,1\% passaram a infringí-la. Já na comercialização de alimentos de transição, 12,7\% deixaram de infringir a NBCAL e 23,6\% passaram a infringí-la. Essas mudanças não alcançaram significância estatística (Tabela 2).

Quanto ao tipo de promoção comercial, na primeira etapa, 143 (92,3\%) (categorias com/sem e com/com) das 155 farmácias apresentaram infração quanto à promoção no preço dos produtos abrangidos pela NBCAL e este percentual foi reduzido para 79,4\% (n = 123) (categorias sem/com e com/com) na segunda etapa. Em relação à exposição especial, 112 farmácias (72,3\%) infringiram a NBCAL na etapa 1, reduzindo para $97(62,6 \%)$ na etapa 2 . Treze farmácias distribuíam brindes ou amostras irregularmente na primeira etapa $(8,3 \%)$ e apenas $3(1,9 \%)$ realizavam esta promoção na segunda etapa. No total de farmácias houve redução significativa para todas as estratégias de promoção comercial irregular (Tabela 3).

Nas farmácias cuja intervenção educativa foi realizada com o farmacêutico, 22\% deixaram de infringir a NBCAL (categoria com/sem) quanto à promoção no preço dos produtos e 4\% passaram a infringir a Norma (categoria sem/com). Nesse mesmo grupo, 26\% deixaram de fazer exposição espe- 
Tabela 2

Frequência de farmácias que infrigiam a Norma Brasileira de Comercialização de Alimentos para Lactentes e Crianças de Primeira Infância, Bicos, Chupetas e Mamadeiras (NBCAL) segundo o tipo de produto em promoção comercial e grupo de alocação, antes e depois da intervenção educativa. Zona Sul da cidade do Rio de Janeiro, Brasil, 2018.

\begin{tabular}{|c|c|c|c|c|c|c|c|c|}
\hline \multirow{3}{*}{$\begin{array}{l}\text { Tipo de produto em promoção } \\
\text { comercial }\end{array}$} & \multicolumn{6}{|c|}{ Grupo de alocação } & \multicolumn{2}{|c|}{ Total } \\
\hline & \multicolumn{2}{|c|}{ GIF } & \multicolumn{2}{|c|}{ GIG } & \multicolumn{2}{|c|}{ GC } & \multirow[b]{2}{*}{$\mathbf{n}$} & \multirow[b]{2}{*}{$\%$} \\
\hline & $\mathbf{n}$ & $\%$ & $\mathbf{n}$ & $\%$ & $\mathbf{n}$ & $\%$ & & \\
\hline \multicolumn{9}{|l|}{$\begin{array}{l}\text { Produtos com promoção comercial } \\
\text { proibida }\end{array}$} \\
\hline Sem/Sem * & 38 & 76,0 & 35 & 76,0 & 46 & 78,0 & 119 & 76,8 \\
\hline Sem/Com ** & 2 & 4,0 & 3 & 6,5 & 2 & 3,4 & 7 & 4,5 \\
\hline Com/Sem *** & 6 & 12,0 & 6 & 13,0 & 4 & 6,8 & 16 & 10,3 \\
\hline Com/Com \# & 4 & 8,0 & 2 & 4,3 & 7 & 11,9 & 13 & 8,4 \\
\hline Total & 50 & 100,0 & 46 & 100,0 & 59 & 100,0 & 155 & 100,0 \\
\hline Valor de $p$ & \multicolumn{2}{|c|}{0,289} & \multicolumn{2}{|c|}{0,508} & \multicolumn{2}{|c|}{0,687} & \multicolumn{2}{|c|}{0,093} \\
\hline \multicolumn{9}{|l|}{ Leites/Compostos lácteos } \\
\hline Sem/Sem * & 2 & 4,0 & 2 & 4,4 & 2 & 3,4 & 6 & 3,9 \\
\hline Sem/Com ** & 2 & 4,0 & 0 & 0 & 3 & 5,1 & 5 & 3,2 \\
\hline 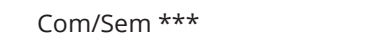 & 11 & 22,0 & 4 & 8,9 & 9 & 15,3 & 24 & 15,6 \\
\hline Com/Com \# & 35 & 70,0 & 39 & 86,7 & 45 & 76,0 & 119 & 77,3 \\
\hline Total & 50 & 100,0 & 45 & 100,0 & 59 & 100,0 & 154 & 100,0 \\
\hline Valor de $p$ & \multicolumn{2}{|c|}{0,022} & \multicolumn{2}{|c|}{0,125} & \multicolumn{2}{|c|}{0,146} & \multicolumn{2}{|c|}{0,001} \\
\hline \multicolumn{9}{|l|}{ Alimentos de transição } \\
\hline Sem/Sem * & 22 & 45,8 & 21 & 47,7 & 24 & 43,6 & 67 & 45,6 \\
\hline Sem/Com ** & 8 & 16,7 & 17 & 38,6 & 13 & 23,6 & 38 & 26,0 \\
\hline 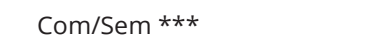 & 7 & 14,6 & 3 & 6,8 & 7 & 12,7 & 17 & 11,6 \\
\hline Com/Com \# & 11 & 22,9 & 3 & 6,8 & 11 & 20,0 & 25 & 17,0 \\
\hline Total & 48 & 100,0 & 44 & 100,0 & 55 & 100,0 & 147 & 100,0 \\
\hline Valor de $p$ & \multicolumn{2}{|c|}{1,000} & \multicolumn{2}{|c|}{0,003} & \multicolumn{2}{|c|}{0,263} & \multicolumn{2}{|c|}{0,006} \\
\hline
\end{tabular}

GC: grupo controle; GIF: grupo de intervenção com o farmacêutico; GIG: grupo de intervenção com o gerente.

* Farmácia não apresentou infração à NBCAL em nenhuma fase da pesquisa;

** Farmácia apresentou infração à NBCAL apenas na segunda fase da pesquisa;

*** Farmácia apresentou infração à NBCAL apenas na primeira fase da pesquisa;

\# Farmácia apresentou infração à NBCAL nas duas fases da pesquisa.

cial de forma irregular e 2\% passaram a fazê-la. Essas reduções apresentaram significância estatistica. Nas farmácias cuja intervenção educativa foi realizada com o gerente, 13\% deixaram de infringir a NBCAL em promoções no preço e 2,2\% passaram a infringi-la. Nesse mesmo grupo, 17,4\% deixaram de fazer exposição especial irregular e 10,9\% passaram a fazê-la, redução não significativa. Nas farmácias do GC, 13,6\% deixaram de realizar promoção no preço dos produtos e 3,4\% passaram a fazer este tipo de promoção, mas esta redução não foi estatisticamente significativa. Não houve alteração na proporção $(15,3 \%)$ de farmácias que deixaram de fazer exposição especial irregular da 1a para a 2a etapa e que passaram a fazê-lo. (Tabela 3 ).

Ao analisar a variação do número de infrações à NBCAL por farmácia, antes e após a intervenção educativa, observou-se no grupo intervenção com o farmacêutico uma variação mediana de -1,5, indicando uma redução no número de infrações à NBCAL por farmácia na segunda etapa em relação à primeira $(\mathrm{p}=0,002)$. Já no grupo de intervenção com o gerente, foi encontrada uma variação mediana de $+0,5$, no entanto, este aumento no número de infrações da primeira para a segunda etapa não foi significativo $(\mathrm{p}=0,680)$. No grupo controle, a variação mediana do número de infrações à NBCAL foi nula e não foi verificada diferença estatisticamente significativa entre as duas etapas no teste de Wilcoxon ( $\mathrm{p}=0,623)$ (Tabela 4). 
Tabela 3

Frequência de farmácias com infração à Norma Brasileira de Comercialização de Alimentos para Lactentes e Crianças de Primeira Infância, Bicos, Chupetas e Mamadeiras (NBCAL), antes e depois da intervenção educativa, segundo a estratégia de promoção comercial e grupo de alocação. Zona Sul da cidade do Rio de Janeiro, Brasil, 2018.

\begin{tabular}{|c|c|c|c|c|c|c|c|c|}
\hline \multirow[t]{3}{*}{ Tipo de promoção } & \multicolumn{6}{|c|}{ Grupo de alocação } & \multicolumn{2}{|c|}{ Total } \\
\hline & \multicolumn{2}{|c|}{ GIF } & \multicolumn{2}{|c|}{ GIG } & \multicolumn{2}{|c|}{ GC } & \multirow[b]{2}{*}{$\mathbf{n}$} & \multirow[b]{2}{*}{$\%$} \\
\hline & $\mathbf{n}$ & $\%$ & $\mathbf{n}$ & $\%$ & $\mathbf{n}$ & $\%$ & & \\
\hline \multicolumn{9}{|l|}{ Desconto no preço } \\
\hline Sem/Sem * & 4 & 8,0 & 1 & 2,2 & 2 & 3,4 & 7 & 4,5 \\
\hline Sem/Com ** & 2 & 4,0 & 1 & 2,2 & 2 & 3,4 & 5 & 3,2 \\
\hline Com/Sem *** & 11 & 22,0 & 6 & 13,0 & 8 & 13,6 & 25 & 16,1 \\
\hline Com/Com \# & 33 & 66,0 & 38 & 82,6 & 47 & 79,7 & 118 & 76,1 \\
\hline Total & 50 & 100,0 & 46 & 100,0 & 59 & 100,0 & 155 & 100,0 \\
\hline Valor de $p$ & \multicolumn{2}{|c|}{0,022} & \multicolumn{2}{|c|}{0,125} & \multicolumn{2}{|c|}{0,109} & \multicolumn{2}{|c|}{$<0,001$} \\
\hline \multicolumn{9}{|l|}{ Exposição especial } \\
\hline Sem/Sem * & 11 & 22,0 & 8 & 17,4 & 9 & 15,3 & 28 & 18,1 \\
\hline Sem/Com ** & 1 & 2,0 & 5 & 10,9 & 9 & 15,3 & 15 & 9,7 \\
\hline Com/Sem *** & 13 & 26,0 & 8 & 17,4 & 9 & 15,3 & 30 & 19,4 \\
\hline Com/Com \# & 25 & 50,0 & 25 & 54,3 & 32 & 54,2 & 82 & 52,9 \\
\hline Total & 50 & 100,0 & 46 & 100,0 & 59 & 100,0 & 155 & 100,0 \\
\hline Valor de $p$ & \multicolumn{2}{|c|}{0,002} & \multicolumn{2}{|c|}{0,581} & \multicolumn{2}{|c|}{1,000} & \multicolumn{2}{|c|}{0,036} \\
\hline \multicolumn{9}{|l|}{ Brindes e amostras } \\
\hline Sem/Sem * & 46 & 92,0 & 42 & 91,3 & 53 & 89,8 & 141 & 90,9 \\
\hline Sem/Com ** & 0 & 0,0 & 0 & 0 & 1 & 1,7 & 1 & 0,6 \\
\hline Com/Sem $* \star \star$ & 4 & 8,0 & 3 & 6,5 & 4 & 6,8 & 11 & 7,1 \\
\hline Com/Com \# & 0 & 0,0 & 1 & 2,2 & 1 & 1,7 & 2 & 1,3 \\
\hline Total & 50 & 100,0 & 46 & 100,0 & 59 & 100,0 & 155 & 100,0 \\
\hline Valor de $p$ & \multicolumn{2}{|c|}{ - } & \multicolumn{2}{|c|}{0,250} & \multicolumn{2}{|c|}{0,375} & \multicolumn{2}{|c|}{0,006} \\
\hline
\end{tabular}

GC: grupo controle; GIF: grupo de intervenção com o farmacêutico; GIG: grupo de intervenção com o gerente.

* Farmácia não apresentou infração à NBCAL em nenhuma fase da pesquisa;

** Farmácia apresentou infração à NBCAL apenas na segunda fase da pesquisa;

*** Farmácia apresentou infração à NBCAL apenas na primeira fase da pesquisa;

\# Farmácia apresentou infração à NBCAL nas duas fases da pesquisa.

\section{Discussão}

A intervenção educativa para a melhoria do cumprimento da NBCAL foi eficaz em alterar as práticas comerciais vigentes, diminuindo o número de farmácias que infringiam a NBCAL e o volume de infrações, principalmente quando o grupo abordado foi o de farmacêuticos. Partiu-se do pressuposto que proporcionar um maior conhecimento sobre uma Norma que foi transformada em Lei Federal há mais de dez anos poderia contribuir para que as promoções comerciais em desacordo com a NBCAL fossem minimizadas. Porém, a redução de infrações à NBCAL ocorreu em uma escala menor do que a esperada, pois as práticas mercantis vigentes são complexas 17 . Os responsáveis tinham pouca autonomia para alterar as infrações à NBCAL frente à imposição de práticas comerciais determinadas pelas redes de estabelecimentos, em um cenário em que a grande maioria deles funciona em rede e é controlada por uma matriz.

O marketing abusivo empreendido por esses estabelecimentos utiliza diferentes vertentes de estímulo ao consumo visando ao lucro e envolvendo interesses de grandes corporações industriais e das redes de farmácias, dificultando a mudança dessas práticas. Burlandy et al. 17 referem a forte influência que as práticas mercadológicas exercem sobre o consumo e remetem às práticas políticas 
Tabela 4

Variação no número de infrações à Norma Brasileira de Comercialização de Alimentos para Lactentes e Crianças de Primeira Infância, Bicos, Chupetas e Mamadeiras (NBCAL) por farmácia antes e após a ação educativa, segundo grupo de alocação. Zona Sul da cidade do Rio de Janeiro, Brasil, 2018.

\begin{tabular}{lccc}
\hline & GIF & GIG & GC \\
\hline Mediana & $-1,5$ & 0,5 & 0,0 \\
DP & 4,497 & 3,912 & 4,520 \\
Amplitude interquartil & 4 & 4 & 3 \\
Mínimo & -19 & -16 & -21 \\
Máximo & 5 & 6 & 17 \\
Valor de p & 0,002 & 0,680 & 0,623
\end{tabular}

DP: desvio padrão; GC: grupo controle; GIF: grupo de intervenção com o farmacêutico; GIG: grupo de intervenção com o gerente.

das grandes corporações alimentícias, que buscam bloquear ou postergar medidas governamentais regulatórias que ferem seus interesses econômicos, como as legislações de proteção à saúde e à alimentação infantil adequada e saudável. Em países em desenvolvimento, o crescimento das vendas dos alimentos infantis ultrapassa $10 \%$ ao ano, já os investimentos na promoção do aleitamento materno têm decrescido globalmente 18 .

As grandes empresas de alimentos e produtos infantis investem fortemente nesses estabelecimentos, por meio das visitas de representantes comerciais que atuam na demonstração dos produtos que competem com o leite materno, na arrumação destes produtos nas prateleiras e em outras estratégias comerciais que buscam gerar uma maior visibilidade e aceitação de seus produtos com vistas à sua aquisição pelos clientes 11 .

Apesar desse cenário desfavorável, foi observada uma redução expressiva no número de infrações à NBCAL nas farmácias cuja intervenção educativa foi realizada com o farmacêutico, e houve um pequeno aumento no número de infrações nas farmácias cuja intervenção educativa foi realizada com o gerente. A intervenção educativa apresentou resultados positivos na redução das promoções comerciais indevidas de fórmulas infantis, mamadeiras, bicos e chupetas (não significativa) e produtos lácteos (significativa), quando realizada com os farmacêuticos, porém o mesmo resultado positivo não foi observado na intervenção educativa realizada com os gerentes. Acredita-se que os farmacêuticos são mais facilmente sensibilizados para o cumprimento da NBCAL, visto que são profissionais da área da saúde e possuem conhecimento a respeito da importância do aleitamento materno 19 .

Os descontos no preço foram a estratégia de promoção comercial mais frequente, seguida das exposições especiais, e a intervenção com o farmacêutico foi mais eficaz em reduzir a frequência destas infrações à NBCAL. Também no México os descontos no preço foram a estratégia de promoção comercial mais frequentemente encontrada em investigação conduzida em 51 pontos de venda 20 , assim como na Indonésia, em pesquisa que envolveu 43 pontos de venda 21 .

O desconhecimento da NBCAL, verificado em mais da metade dos gerentes e farmacêuticos entrevistados nesta pesquisa, foi consistente com outros estudos. Outra investigação realizada na Zona Sul do Rio de Janeiro 11, em 2017, observou que 55,7\% dos responsáveis por farmácias não conheciam a NBCAL, e um estudo realizado no ano 20009 em 139 farmácias localizadas em diferentes municípios brasileiros verificou que $89,9 \%$ dos funcionários não conheciam a NBCAL.

Apesar da promoção comercial de fórmulas infantis para lactentes, mamadeiras e bicos estar proibida no Brasil há três décadas 9,10, e da promoção comercial de leites e alimentos de transição ser permitida apenas quando acompanhada de frase de advertência 10, mais de dois terços das farmácias comercializavam estes produtos de forma ilegal, infringindo a NBCAL.

Após a intervenção educativa, foi observada uma redução no percentual de farmácias com infração à NBCAL na maioria dos grupos de alimentos e de produtos infantis, abrangidos pela Lei no 
11.265/2006, com exceção dos alimentos de transição. Esse aumento no percentual de infrações nos alimentos de transição foi observado tanto nos grupos intervenção quanto no grupo controle, sugerindo que o aumento pode ter ocorrido devido a algum fator contextual da época da segunda etapa da pesquisa, como uma maior disponibilidade desses produtos no mercado, o que não teve relação com a intervenção educativa.

Essa expressiva proporção de estabelecimentos infringindo a NBCAL na comercialização de alimentos de transição também foi verificada em um estudo 22 realizado em 2016 em supermercados de Mossoró (Rio Grande do Norte), onde $100 \%$ da amostra selecionada $(\mathrm{n}=25)$ infringiam a NBCAL, principalmente na comercialização inadequada de alimentos de transição (64\%). Também em um estudo realizado na Indonésia 21 as promoções dos alimentos de transição foram frequentes, observadas em $81 \%$ dos estabelecimentos que os comercializavam, principalmente naqueles maiores.

Uma pesquisa recente 23 sobre o cumprimento da NBCAL em sites dos fabricantes e redes de farmácias, identificou que a infração mais recorrente nos sites dos fabricantes de alimentos infantis foi a ausência de frases de advertência em destaque na promoção comercial de produtos destinados a crianças com mais de seis meses de idade, e que apenas 33\% dos sites de redes de farmácias cumpriram a Lei no 11.265/2006. Pode haver maior dificuldade por parte dos responsáveis por farmácias em perceber o efeito negativo da introdução dos alimentos de transição sobre a prática da amamentação, por serem estes produtos indicados para crianças maiores de seis meses. No entanto, apesar de contraindicada, sua introdução na dieta infantil costuma ser precoce, ainda no primeiro semestre de vida, prejudicando a saúde e a nutrição infantil 24 . Alguns alimentos introduzidos precocemente na dieta da criança, como os cereais infantis, costumam ter alto teor de açúcar e são frequentemente misturados ao leite para engrossá-lo. Uma análise das refeições lácteas consumidas por crianças entre 6 e 23 meses de idade, usuárias do Sistema Único de Saúde (SUS) no Município do Rio de Janeiro, revelou a presença de espessante e/ou açúcar e/ou fruta em $86,9 \%$ das preparações, conferindo excesso de ingestão de energia, contribuindo para a obesidade infantil 25.

A ausência de monitoramento regular e de ações educativas sobre a Lei no 11.265/2006 impacta fortemente o atual cenário de descumprimento da NBCAL 26. Medidas de proteção ao aleitamento materno precisam ser tomadas pelos órgãos competentes para evitar o marketing abusivo desses produtos que competem com a amamentação. As punições cabíveis em Lei devem ser aplicadas aos estabelecimentos comerciais que infringem a NBCAL, como advertência, multa, apreensão do produto e interdição parcial ou total do estabelecimento 27.

Dentre as limitações do presente estudo, podemos referir o pequeno lapso de tempo entre as duas etapas da pesquisa, que pode ter sido insuficiente para que seus responsáveis implementassem as mudanças necessárias nas práticas comerciais, como a colocação das frases de advertência preconizadas 10, o que pode ter contribuído para uma menor eficácia da intervenção educativa. Outro aspecto que pode ter ocorrido foi a contaminação do grupo controle pela entrevista ao responsável, realizada na primeira etapa da pesquisa, quando este era questionado sobre o conhecimento da NBCAL, já que algumas promoções comerciais indevidas reduziram também no grupo controle. A participação de farmácias do grupo controle integradas a uma rede, da qual participavam também farmácias do grupo intervenção, pode também ter contribuído para uma possível contaminação do grupo controle. Essas limitações podem ter levado à subestimação do efeito da ação educativa.

Conclui-se que uma intervenção educativa realizada com farmacêuticos foi capaz de melhorar de forma significativa o cumprimento da NBCAL nas farmácias. A orientação a respeito da Lei no 11.265/2006 pareceu aumentar a sensibilização dos farmacêuticos sobre o seu papel na proteção do aleitamento materno e instrumentalizá-los no cumprimento da NBCAL. O farmacêutico, como profissional de saúde, com base em seu conhecimento e em seus princípios éticos, pareceu ter mais capacidade de se contrapor às pressões da indústria, desempenhando papel estratégico para o cumprimento da NBCAL.

Recomenda-se intensificar as ações de fiscalização das práticas comerciais relativas aos alimentos infantis, mamadeiras, bicos e chupetas, bem como a ampliação do conhecimento dessa legislação por outros atores, como os responsáveis pelas redes de farmácias, os profissionais de saúde e as mães, para que essa importante legislação conquistada no Brasil possa proteger efetivamente o aleitamento materno e a saúde infantil. 


\section{Colaboradores}

G. P. N. Rodrigues foi responsável pela concepção e planejamento do estudo e contribuiu na análise e interpretação dos dados, revisão bibliográfica, discussão dos resultados e redação do manuscrito. M. I. C. Oliveira contribuiu na concepção e planejamento do estudo, análise e interpretação dos dados, revisão bibliográfica, discussão dos resultados e redação do manuscrito. C. S. Boccolini contribuiu na análise e interpretação dos dados, discussão dos resultados e redação do manuscrito. E. O. F. Sally contribuiu na concepção e planejamento do estudo, interpretação dos dados, discussão dos resultados e redação do manuscrito. J. R. Moraes contribuiu na amostragem, análise e interpretação dos dados, discussão dos resultados e redação do manuscrito.

\section{Informações adicionais}

ORCID: Gloria Priscila Nunes Rodrigues (00000002-9326-2426); Maria Inês Couto de Oliveira (0000-0003-0439-6295); Cristiano Siqueira Boccolini (0000-0002-4804-5641); Enilce de Oliveira Fonseca Sally (0000-0002-9506-3690); José Rodrigo de Moraes (0000-0003-4814-5076).

\section{Agradecimento}

Ao Conselho Nacional de Desenvolvimento Científico e Tecnológico (CNPq; processo no 404948/2016-2).

\section{Referências}

1. World Health Organization. Indicators for assessing infant and young child feeding practices. https://www.who.int/nutrition/publi cations/infantfeeding/9789241599290/en/ (acessado em 31/Jul/2020).

2. Victora CG, Bahl R, Barros AJ, França GV, Horton S, Krasevec J, et al. Breastfeeding in the 21st century: epidemiology, mechanisms, and lifelong effect. Lancet 2016; 387:475-90.

3. Boccolini CS, Boccolini PMM, Monteiro FR, Venâncio SI, Giugliani ERJ. Breastfeeding indicators trends in Brazil for three decades. Rev Saúde Pública 2017; 51:108.

4. Boccolini CS, Carvalho ML, Oliveira MIC. Factors associated with exclusive breastfeeding in the first six months of life in Brazil: a systematic review. Rev Saúde Pública 2015; 49:240-8.

5. Rollins NC, Bhandari N, Hajeebhoy N, Horton S, Lutter CK, Martines JC, et al. Why invest, and what it will take to improve breastfeeding practices? Lancet 2016; 387:491-504.

6. Hernandez AR, Victora CG. Biopolíticas do aleitamento materno: uma análise dos movimentos global e local e suas articulações com os discursos do desenvolvimento social. Cad Saúde Pública 2018; 34:e00155117.

7. World Health Organization; United Nations Children's Fund. International code of marketing of breast-milk substitutes. https://www. who.int/nutrition/publications/code_english. pdf (acessado em 03/Mar/2021).

8. Departamento de Ações Programáticas Estratégicas, Secretaria de Atenção à Saúde, Ministério da Saúde. Bases para a discussão da Política Nacional de Promoção, Proteção e Apoio ao Aleitamento Materno. Brasília: Ministério da Saúde; 2017.

9. Cyrillo DC, Sarti FM, Farina EMQ, Mazzon JA. Duas décadas da Norma Brasileira de Comercialização de Alimentos para Lactentes: há motivos para comemorar? Rev Panam Salud Pública 2009; 25:134-40.

10. Brasil. Lei no 11.265 , de 3 de janeiro de 2006. Regulamenta a comercialização de alimentos para lactentes e crianças de primeira infância e também a de produtos de puericultura correlatos. Diário Oficial da União 2006; 4 jan.

11. Silva KB, Oliveira MIC, Boccolini CS, Sally EOF. Illegal commercial promotion of products competing with breastfeeding. Rev Saúde Pública 2020; 54:10.

12. Brousselle A, Champagne F, Contandriopoulos A-P, Hartz Z, organizadores. Avaliação: conceitos e métodos. Rio de Janeiro: Editora Fiocruz; 2011.

13. Hájek J. Limiting distribution in simple random sampling from a finite population. Publications of the Mathematics Institute of the Hungarian Academy of Science 1960; 5:36174. 
14. World Health Organization. NetCode toolkit. Monitoring the marketing of breast-milk substitutes: protocol for ongoing monitoring systems. Geneva: World Health Organization; 2017.

15. Abbot A. Paper, paper, everywhere... Nature 2005; 437:310.

16. Siegel S, Castellan NJ. Estatística não paramétrica para ciências do comportamento. 2a Ed. Porto Alegre: Artmed; 2006.

17. Burlandy L, Alexandre VP, Gomes FS, Castro IRR, Dias PC, Henriques P, et al. Políticas de promoção da saúde e potenciais conflitos de interesses que envolvem o setor privado comercial. Ciênc Saúde Colet 2016; 21:1809-18.

18. Piwoz EG, Huffman SL. The impact of marketing of breast-milk substitutes on WHOrecommended breastfeeding practices. Food Nutr Bull 2015; 36:373-86.

19. Silva LIMM, Thé PMP, Medeiros JO, Giacomini SGMO, Rodrigues RCB, Peixoto MMLV. Conhecimento de farmacêuticos sobre aleitamento materno: um estudo nas farmácias comerciais em Fortaleza - CE. Rev Bras Promoç Saúde 2012; 25:482-91.

20. Hernández-Cordero S, Lozada-Tequeanes AL, Shamah-Levy T, Lutter C, Cosío TG, SaturnoHernández P, et al. Violations of the International Code of Marketing of Breast-milk Substitutes in Mexico. Matern Child Nutr 2019; 15:e12682.

21. Hadihardjono DN, Green M, Stormer A, Agustino, Izwardy D, Champeny M. Promotions of breastmilk substitutes, commercial complementary foods and commercial snack products commonly fed to young children are frequently found in points-of-sale in Bandung City, Indonesia. Matern Child Nutr 2019; 15 Suppl 4:e12808.
22. Gurgel TEP. Monitoramento da promoção comercial de alimentos para lactentes e crianças de primeira infância e de produtos de puericultura em estabelecimentos comerciais de Mossoró. Nutrivisa - Revista de Nutrição e Vigilância em Saúde 2016; 1:21-5.

23. Prado ISCF, Rinaldi AEM. Compliance of infant formula promotion on websites of Brazilian manufacturers and drugstores. Rev Saúde Pública 2020; 54:12.

24. Ministério da Saúde. Pesquisa Nacional de Demografia e Saúde da Criança e da Mulher. Brasília: Ministério da Saúde; 2008.

25. Furtado MCQ, Castro IRR, Silva ACF, Moraes MM. Preparações lácteas consumidas na alimentação complementar: caracterização e composição nutricional segundo a participação de alimentos ultraprocessados. Demetra (Rio J.) 2019; 14 Suppl 1:e43765.

26. Instituto Brasileiro de Defesa do Consumidor. Pesquisa Amamentação: amamentação desvalorizada. Revista do IDEC 2015; fev. http:// www.idec.org.br/uploads/revistas_materias/ pdfs/195-amamentacao1.pdf.

27. Brasil. Lei no 6.437, de 20 de agosto de 1977. Configura infrações à legislação sanitária federal, estabelece as sanções respectivas, e dá outras providências. Diário Oficial da União 1977; 24 ago. 


\section{Abstract}

The Brazilian Code of Marketing of Infant and Toddlers Food, Nipples, Pacifiers, and Baby Bottles (Federal Law n. 11,265/2006) aims to control the abusive marketing of products that compete with breastfeeding. The objective was to assess the impact of an educational intervention on compliance with this law by pharmacies. A randomized intervention study was conducted in 155 pharmacies that were infringing the law in the Southern Zone of Rio de Janeiro, Brazil. The pharmacies' commercial practices were assessed before and after the intervention with the pharmacist and manager, compared to the control group. The interval between the intervention and the second assessment was one month. McNemar test was used to measure changes in the number of pharmacies infringing the law before and after the intervention. Wilcoxon test was used to compare variation in the number of violations in each group. A month after the intervention, there was a $16.1 \%$ reduction $(n=25)$ in the number of pharmacies committing violations. There was a decrease from $18.7 \%$ to $12.9 \%$ in products whose commercial promotion is banned by the law ( $p=$ 0.093 ) and from $92.9 \%$ to $80.5 \%$ in milk products $(p=0.001)$, but among processed complementary food there was an increase from $28.5 \%$ to $42.3 \%$ of pharmacies with illegal promotions $(p=0.006)$. The intervention group with the pharmacists showed a statistically significant reduction in violations related to discount prices $(p=0.022)$ and special displays $(p=0.002)$. The educational intervention reduced the number of pharmacies that infringed the law, mainly when the intervention involved the pharmacist.

Experimental Studies; Breast Feeding; Food Legislation; Marketing; Breast-Milk Substitutes

\section{Resumen}

La Norma Brasileña de Comercialización de Alimentos para Lactantes y Niños de la Primera Infancia, Tetillas, Chupetes y Biberones (Ley Federal no 11.265/2006) tiene como finalidad controlar el márketing abusivo de productos que compiten con la lactancia materna. El objetivo fue evaluar el impacto de una intervención educativa sobre el cumplimiento de la ley en farmacias. Se realizó una investigación de intervención aleatoria, llevada a cabo en 155 farmacias que infringían la ley en la zona sur de Río de Janeiro, Brasil. La práctica comercial de las farmacias fue evaluada antes y después de la intervención con el farmacéutico y con el gerente, comparadas con el grupo de control. El intervalo entre la intervención y la segunda evaluación fue de un mes. Para evaluar los cambios en lo cuantitativo de farmacias con infracción a la ley, antes y después de la intervención, se utilizó el test de McNemar. El test de Wilcoxon se utilizó para comparar la variación en el número de infracciones en cada grupo de asignación. Tras un mes de la intervención hubo una reducción de un $16,1 \%(n=25)$ en el total de farmacias con infracción. Hubo una reducción desde un $18,7 \%$ a un $12,9 \%$ en productos cuya promoción comercial está prohibida $(p=0,093)$ y de un $92,9 \%$ a un $80,5 \%$ en productos lácteos $(p=0,001)$. Sin embargo, entre alimentos de transición hubo un aumento de un 28,5\% a un 42,3\% de farmacias con promoción ilegal $(p=0,006)$. El grupo de intervención con el farmacéutico presentó una reducción estadísticamente significativa en las infracciones relacionadas con descuentos de precio ( $p$ $=0,022)$ y respecto a exposiciones especiales $(p=$ 0,002 ). La intervención educativa redujo la cantidad de farmacias que infringía esta ley, principalmente cuando la intervención educativa se realizó con el farmacéutico.

Estudios Experimentales; Lactancia Materna;

Legislación Alimentaria; Mercadotecnía;

Sustitutos de la Leche Humana
Recebido em 06/Mar/2020

Versão final reapresentada em 13/Ago/2020 Aprovado em 20/Ago/2020 